\title{
LAS PLANTAS COMESTIBLES EMPLEADAS POR LAS comunidades comechingonas de SAN Marcos Sierras (Córdoba, Argentina). Primeras aproximaciones
}

\author{
Edible plants used by Comechingon communities from San Marcos \\ Sierras (Córdoba, Argentina). A first approach
}

\author{
Valentina Saur Palmieri ${ }^{1 *}$ y Melisa G. Geisa ${ }^{2}$ (D)
}

1. Departamento de Diversidad Biológica y Ecológica, Facultad de Ciencias Exactas, Físicas y Naturales, Universidad Nacional de Córdoba - CONICET, Argentina.

2. Instituto de Antropología de Córdoba (IDACOR), Universidad Nacional de Córdoba - CONICET, Córdoba, Argentina.

\section{*altea345@hotmail.com}

\section{Citar este artículo}

Saur Palmieri, V. \& M. G. Geisa. 2019. Las plantas comestibles empleadas por las comunidades comechingonas de San Marcos Sierras (Córdoba, Argentina). Primeras aproximaciones. Bol. Soc. Argent. Bot. 54: 295-309.

DOI: http://dx.doi. org/10.31055/1851.2372.v54. n2.24374

Recibido: 23 Enero 2019 Aceptado: 14 Abril 2019 Publicado: 30 Junio 2019 Editora: Norma Hilgert (D)

\section{SUMMARY}

Background and aims: Since the 1990s in Argentina, a process of visibility and reemergence of aboriginal groups that were believed extinct by official history has been developing. This is the case of Comechingon people in Córdoba province. Furthermore, it is known that gathering of wild food plants is an ancient practice that contributes to the subsistence of many indigenous communities of the country. Within the framework of Food Sovereignty and Ethnobiology, the aim of this paper was to carry out a first inquiry into the use of wild edible plants by residents self-identified as members of Comechingon people of San Marcos Sierras (Córdoba).

M\&M: Semi-structured interviews and free listings were conducted to a representative from each of three Comechingon communities from this town. They were accompanied with the discussion of a photographic guide of species.

Results: Thirty-four wild ethnotaxa were recognized. They correspond to thirty-two botanical taxa. $85 \%$ was native species and the rest was exotic. "Algarrobo trees" (Prosopis spp.) presented the greatest number of parts used and ways of consumption mentioned. Fruits were the most used vegetable parts, followed by flowers, leaves, cladodes, underground organs and resins. The last two, just like trees' leaves, had not been previously reported for the region. Likewise, different ways of gathering, conservation and culinary products were mentioned.

Conclusions: Comechingon communities know and consume a wide variety of edible ethnotaxa of plants. We hope to contribute to the visibility of Comechingon culture and the consolidation of sovereign food systems.

\section{KeY WORDS}

Chaco serrano, Comechingón people, edible plants, ethnobiology, food sovereignty.

\section{RESUMEN}

Introducción y objetivos: Desde la década de 1990, en Argentina se aprecia un proceso de visibilización y resurgimiento de grupos aborígenes que se creían extintos por parte de la historia oficial. Este es el caso del Pueblo Comechingón en la provincia de Córdoba. En otro sentido, se sabe que la recolección de plantas silvestres alimenticias es una práctica ancestral que contribuye a la subsistencia de muchas comunidades indígenas del país. En el marco de la soberanía alimentaria y la etnobiología, se planteó realizar una primera indagación sobre el uso de plantas silvestres comestibles por parte de pobladores que se auto-identifican pertenecientes al pueblo comechingón de San Marcos Sierras (Córdoba).

M\&M: Se realizaron entrevistas semiestructuradas, listados libres y la observación de un muestrario fotográfico guía, a un referente en tres comunidades comechingonas de la localidad.

Resultados: Los entrevistados reconocieron 34 etnoespecies silvestres, correspondientes a 32 taxones botánicos. El 85\% fueron nativas y el resto exóticas. Los "algarrobos" (Prosopis spp.) presentaron la mayor cantidad de partes utilizadas y formas de consumo registradas. Las estructuras vegetales más citadas fueron los frutos, seguido de las flores, hojas, cladodios, órganos subterráneos y resinas. Estas dos últimas, junto con las hojas de árboles, no habían sido reportadas anteriormente para la región. Asimismo, se aludió a diferentes modos de colecta, conservación y productos culinarios.

Conclusiones: Las comunidades comechingonas conocen y consumen una amplia variedad de plantas comestibles. Se espera contribuir a la visibilización de la cultura comechingón y a la consolidación de sistemas de alimentación soberanos.

\section{Palabras clave}

Chaco serrano, etnobiología, plantas alimenticias, Pueblo Comechingón, soberanía alimentaria. 


\section{INTRODUCCIÓN}

La importancia de estudiar los vegetales comestibles en comunidades re-identificadas como originarias

Desde los años 1990 en Argentina, se puede visualizar la emergencia de "nuevas identidades" producto de procesos sociales de identificación étnica y la organización de grupos indígenas que se creían extintos o en vías de extinción (Stagnaro, 2016). Esto es lo que Bartolomé denomina "Etnogénesis" (Bartolomé, 1997). Esta re-identificación con lo aborigen (sensu Briones, 1998), se produciría en respuesta a la invisibilización (sensu Valverde, 2013) sufrida por los pobladores originarios y a la reivindicación de lo indígena que se sucede en toda Latinoamérica desde finales del siglo XX (Stagnaro, 2009). En el caso del territorio argentino Bompadre (2013) señala que las movilizaciones de esta índole fueron llevadas a cabo por diversos grupos en repudio a las celebraciones en torno al "Quinto Centenario" es decir, el sentido de festejo otorgado por el Estados al cumplimiento de 500 años de conquista y colonización europea. Otra de las razones de la organización de estos grupos, fue la motivación de participar en las discusiones en torno a la reforma constitucional de 1994 que culminaron en el "reconocimiento de derechos especiales para los que se declaran como parte de los pueblos indígenas preexistentes al estado nacional" (Stagnaro, 2016). De esta manera, se instauró la auto-adscripción identitaria (auto-afirmación de una persona como indígena dentro de una comunidad) y el otorgamiento de personerías jurídicas a las comunidades que lo soliciten (Ministerio de Educación \& UNICEF, 2010; Stagnaro, 2016).

Las reconstrucciones del pasado indígena de la provincia de Córdoba, en particular del llamado Período Prehispánico Tardío que transcurre entre el 1500 y el $300 \mathrm{AP}$, realizadas a partir de diversos trabajos de prospección y excavación arqueológica han permitido la caracterización de los pobladores prehispánicos del centro de Argentina (Pastor et al., 2012). De esta manera, se considera que los grupos humanos poseían una alta movilidad residencial, ocupaban diversos paisajes en el ambiente de forma estacional y aprovechaban todos los recursos disponibles (Medina et al., 2016). En referencia al sistema de subsistencia, la economía era mixta y se basaba en recursos silvestres, tanto vegetales como animales. Es decir que, aunque practicaban una agricultura a pequeña escala, sin tecnificación y secano, la recolección era una actividad de gran importancia (Pastor \& López, 2011; López, 2015). Con la llegada de los españoles al actual territorio cordobés en el siglo XVI, los pueblos originarios fueron sometidos a las encomiendas, al trabajo y evangelización en las misiones jesuíticas, desplazados de los territorios donde habitaban hacia zonas más desfavorables, y transformando progresivamente su cultura. Dicha desarticulación de las comunidades continuó y se reforzó en el siglo XIX con la conformación del Estado Nacional junto a una "identidad nacional" asociada, donde "lo indio" era marginado y denostado. Todo esto contribuyó a la generalización de la "narrativa de la extinción" de los indígenas, es decir a la creencia establecida de la desaparición total de los pobladores originarios de la actual Provincia de Córdoba (Stagnaro, 2016). Actualmente, el proceso colectivo de visibilización, reivindicación cultural y auto-reconocimiento mencionado anteriormente, confronta con el "relato histórico oficial" que continúa pregonando la inexistencia indígena y tilda de ilegítimos a los ciudadanos que eligen dicha identidad (Bompadre, 2013; Stagnaro, 2016). En los últimos años, varios cordobeses comenzaron a reconstruir el pasado personal y colectivo a partir de la recuperación de la memoria familiar y de investigaciones de documentación histórica llevadas a cabo por ellos mismos (Palladino, 2013; Stagnaro, 2016).

En particular en este trabajo, centraremos el análisis en el Pueblo Comechingón. La identificación de este pueblo con dicha denominación proviene del proceso de conquista y colonización europea; es decir, los españoles nombraron de esta forma a un conjunto de pueblos de similares características lingüísticas y formas de organización social y política que habrían ocupado el área de las sierras centrales de la actual provincia de Córdoba y San Luis. A pesar de que el origen del término y su significación todavía se encuentran en debate, algunas especulaciones sugieren que podría tratarse de un grito de guerra o hacer referencia a los lugares que habitaban los antiguos pobladores de la región (Gleser, 2009; Stagnaro, 2016; Tulián, 2016). En este sentido, y aunque se plantea que "el nombrar" respondía a los intereses 


\section{Saur Palmieri y M. G. Geisa - Plantas comestibles en comunidades comechingonas}

de los conquistadores, resulta interesante que este nombre haya sido resignificado positivamente por quienes se identifican como parte de un pueblo originario (Stagnaro, 2016). No obstante, la invisibilización sufrida por siglos y en coherencia con la re-etnización (sensu Bompadre, 2013), en el presente en la Provincia de Córdoba existen más de 5000 personas que se reconocen pertenecientes y/o son descendientes en primera generación del Pueblo Comechingón (INDEC, 2004). Una de las localidades cordobesas donde existen comunidades que se auto-reconocen comechingonas es San Marcos Sierras, ubicada en el sector noroeste de la provincia. En esta localidad desarrollamos el presente estudio.

\section{Las plantas comestibles en la cultura de los pueblos}

La recolección de plantas silvestres alimenticias es una costumbre muy arraigada culturalmente y de gran importancia no sólo en grupos cazadoresrecolectores sino también en sociedades que dependen de la agricultura (Arias Toledo, 2008). Esta práctica ancestral contribuyó y, aún en la actualidad, contribuye a la subsistencia de muchas comunidades aborígenes de nuestro país (Rapoport et al., 1998; Ladio \& Lozada, 2000; Azar, 2002; Arenas, 2003; Ladio, 2004; Scarpa, 2009; Aguirre, 2012; Aldazabal et al., 2012; Martínez, 2012; Martínez Crovetto, 2012; Montani \& Scarpa, 2016). Asimismo, otros sectores sociales, campesinos y suburbanos de diversas regiones también conocen y utilizan vegetales silvestres, aunque no basan su alimentación en estos ítems (Hilgert, 1999; Ladio \& Rapoport, 1999; Ladio, 2005; Biurrun et al., 2007; Ochoa \& Ladio, 2011; Muiño, 2012; Hurrell et al., 2013).

La percepción, importancia y utilización efectiva de plantas útiles está relacionada con factores ecológicos y culturales (Biurrun et al., 2007 y bibliografía allí citada). Entre estos últimos, podemos mencionar el contexto histórico y el sistema de creencias que un grupo social posee. En este sentido, diversos procesos como la industrialización de la comida, la expulsión de población rural a las grandes ciudades y la subestimación del uso de alimentos silvestres, así como también los procesos de degradación de áreas naturales han producido que la práctica de recolectar plantas para la ingesta haya perdido relevancia (Ladio \& Rapoport, 1999; Biurrun et al., 2007; Arias Toledo, 2008).
En la provincia de Córdoba, ya a principios del siglo XX se daba cuenta de 20 especies comestibles y diversos productos obtenidos de las mismas (Río \& Achával, 1905). En la actualidad, diversos trabajos etnobotánicos documentan el uso de la mayoría de estas especies (Arias Toledo et al., 2007a; Arias Toledo, 2008; Trillo et al., 2014; Trillo, 2016). Sin embargo, las investigaciones mencionadas existentes se han realizados con pobladores rurales que se autodenominan "criollos" (Trillo, 2010). Debido a esto último, y en el marco del proceso de emergencia étnica aborigen que está ocurriendo en Córdoba, en este trabajo indagamos sobre el uso de plantas nativas comestibles por parte de pobladores que se auto-identifican como pertenecientes al Pueblo Comechingón.

En este sentido, en el presente estudio tomamos como marco conceptual la noción de "Soberanía Alimentaria", definida como "el derecho de los pueblos a alimentos nutritivos y culturalmente adecuados, accesibles, producidos de forma sostenible y ecológica, y su derecho a decidir su propio sistema alimentario y productivo" (Rosset, 2003; Foro Mundial de la Soberanía Alimentaria, 2007). El concepto de Soberanía Alimentaria, surge de las organizaciones campesinas a nivel mundial e implica otras epistemologías (De Sousa Santos, 2010), al involucrar formas de construcción de conocimiento alternativas, con un fuerte anclaje territorial. Por otro lado, aunque esta conceptualización está vinculada usualmente a la práctica agrícola -es decir el cultivo de alimentos-, en el presente trabajo entendemos la práctica de recolección de frutos silvestres, como una tarea indisociable de la alimentación soberana, asociada a conocimientos ancestrales y al manejo de los ecosistemas naturales.

En suma, consideramos a la Soberanía Alimentaria como un anclaje interesante para revalorizar saberes ancestrales de las comunidades y ponerlos en juego en los procesos de reidentificación y defensa territorial que vienen llevando a cabo diferentes sectores sociales, entre ellos los pueblos originarios.

Por lo dicho anteriormente, el objetivo de este trabajo es realizar un aporte preliminar a la identificación de las plantas nativas reconocidas como alimenticias por los pobladores de San Marcos Sierras que se reconocen como pertenecientes y son representantes de cada comunidad del Pueblo 
Comechingón, sus usos, formas de consumo y las prácticas asociadas. Entendemos la revalorización de estos saberes como una primera contribución a las discusiones acerca de la necesidad de considerar a los vegetales silvestres dentro del marco de la Soberanía Alimentaria.

\section{Materiales y Métodos}

\section{Área de Estudio}

Características ambientales: La localidad de San Marcos Sierras (3046'58.0" S, 64³8'10.7" W) se ubica $139 \mathrm{~km}$ al noroeste de la capital de la Provincia de Córdoba, en el departamento Cruz del Eje (Fig. 1). Pertenece a la región fitogeográfica Chaqueña, distrito Chaco Serrano (Cabrera, 1976), que se caracteriza por poseer un bosque xerófilo a sub xerófilo dominado por Schinopsis lorentzii (Griseb.) Engl. y Lithraea molleoides (Vell.) Engl., con variadas especies de los géneros Baccharis, Eupatorium, Euphorbia, Gymnocalycium y Tillandsia (Giorgis et al., 2011). En la provincia, el Distrito Chaqueño Serrano presenta una amplia diversidad vegetal representada por variadas formas de vida $(\mathrm{n}=17)$, incluye endemismos y alberga cerca del 50\% de especies citadas para Córdoba (Giorgis et al., 2011).

Características históricas y socioculturales: En el siglo XVI, en el momento de la llegada de los españoles, la zona de San Marcos Sierras estaba habitada por 24 pueblos y se conocía como Tay Pichín, Tay Pitín o Tulián cabiche. En 1573 fue otorgada en encomienda, la cual se disolvió en 1806 durante el comisionado del Virrey Marqués de Sobremonte. Esto se consiguió luego de una larga demanda de los pueblos originarios representados por el Cacique Francisco Tulián, proceso que culminó con la devolución de las tierras (ADIC, 2000; Tulián, 2016). Más tarde, entre 1892 y 1895, el gobierno de la Provincia de Córdoba se apropia del territorio de la comunidad y realiza un loteo ignorando los reclamos de los habitantes originarios de la región (Tulián \& Palladino, 2015). Con el transcurso del tiempo la localidad adquirió heterogeneidad cultural, fruto de la coexistencia de vertientes poblacionales que incluyen los pueblos originarios, los descendientes de españoles de la época colonial, los "naturistas" que arribaron a la región en la década de 1930 y migrantes de

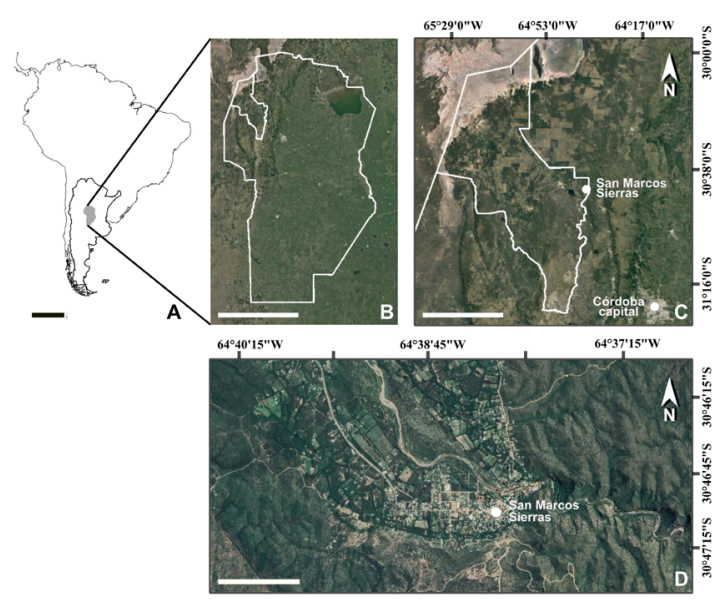

Fig. 1. Ubicación del área de estudio. A: Argentina, Provincia de Córdoba. B: Provincia de Córdoba, Dpto. Cruz del Eje. C: Dpto. Cruz del Eje, Localidad de San Marcos Sierras. D: Imagen Satelital de la localidad de San Marcos Sierras. Escalas= A: 1000 km; B: 200 km, C: 50 km, D: $1 \mathrm{~km}$.

distintos lugares del país (Santa Fe y Buenos Aires, principalmente) y de otros países como Alemania, Francia y Estados Unidos (Municipalidad de San Marcos Sierras \& Universidad Nacional de Córdoba, 2009). En el último censo disponible contaba con 2199 habitantes (INDEC, 2010). Las actividades económicas preponderantes son el turismo y la apicultura (Municipalidad de San Marcos Sierras \& Universidad Nacional de Córdoba, 2009).

Con respecto al pasado originario, existen algunos intentos formales de rescatar los orígenes de la cultura ancestral y la preservación del patrimonio cultural Comechingón - Sanavirón (Ej.: Ordenanza de Planeación y Ordenamiento Territorial $\mathrm{N}^{\circ}$ 596/09; Municipalidad de San Marcos Sierras \& Universidad Nacional de Córdoba, 2009). En este sentido, aunque no exenta de críticas por parte de las comunidades indígenas, la Ley Provincial $\mathrm{N}^{\circ}$ 10.308 crea la Reserva Arqueológica Quilpo que reconoce más de 20 sitios arqueológicos en la zona (Ley $\left.\mathrm{N}^{\circ} 10.308,2015\right)$. Los habitantes conocen que muchos otros sitios han sido desmantelados en el pasado (Tulián \& Palladino, 2015).

Producto de los procesos de re-identificación mencionados en la introducción, en el municipio al momento de realizar el estudio se reconocían 


\section{Saur Palmieri y M. G. Geisa - Plantas comestibles en comunidades comechingonas}

tres comunidades comechingonas Tulián, Tacu Kuntur y Orko Huasi; luego por observaciones personales evidenciamos que se conformó una nueva comunidad denominada "Tay Pichín”. Las tres comunidades involucradas en el estudio, cuentan con reconocimiento oficial. La Comunidad Territorial Comechingón Sanavirón Tulián y Tacu Kuntur están reconocidas formalmente frente al INAI (Res. INAI $\mathrm{N}^{\circ} 064 / 10$ y Res. INAI ${ }^{\circ}$ $144 / 09$, respectivamente) y cuentan con sus propios estatutos, mientras que Orko Huasi se encuentra avalada por el Consejo Educativo Autónomo de los Pueblos Indígenas de Córdoba según ha comunicado el referente de esa comunidad entrevistado a las autoras de este trabajo. Sus integrantes se comenzaron a reunir en los años 90' para reflotar las prácticas de sus antepasados. En estos tiempos, se comenzaba a realizar una fiesta denominada "La Tulianada" que funcionaba como espacio común de encuentro y reunión, y abrazaba un "sentimiento de enorgullecimiento de las raíces". Luego de la creación de ese espacio que nucleaba a todas esas personas, con el tiempo se formaron las comunidades existentes en la actualidad. El espacio de La Tulianada fue manipulado con motivos ajenos a las comunidades originarias y en el presente éstas no se sienten representadas con este evento, que se mantiene como un atractivo turístico de la Municipalidad (Comunicado de la Comunidad Tulián frente a la organización de La Tulianada del 16 de marzo de 2017; Cultura San Marcos Sierras, 2017).

\section{Obtención de información y análisis de datos}

En mayo de 2017, se entrevistó en una oportunidad a un miembro de cada comunidad propuesto por el mismo grupo para colaborar con el presente estudio. Se entrevistó a Raúl Verasay (R.V.; Casqui curaca ${ }^{1}$ de la Comunidad Orko Huasi), Oscar Eduardo "Meliño" Tulián (O.E.T.; representante sugerido por la Casqui curaca de la Comunidad Tulián) y Leopoldo Tulián (Casqui curaca de la Comunidad Tacu Kuntur). En estos encuentros se combinaron distintas técnicas etnográficas: entrevista semiestructurada (Bernard, 1995), listado libre (Cotton, 1996) y la observación y discusión de un muestrario fotográfico guía

\footnotetext{
El concepto de "Casqui Curaca" refiere al líder de la comunidad.
}

confeccionado a partir de una revisión bibliográfica previa sobre las especies comestibles presentes en el área (Di Lullo, 1935; Biurrun et al., 2007; Arias Toledo, 2008; Rapoport et al., 2009; Giorgis et al., 2011; Trillo et al., 2014; Trillo, 2016). Las entrevistas se registraron en grabador digital y en planilla de campo, con consentimiento escrito informado previamente, según lo propuesto en por Cano-Contreras et al. (2016). Los vegetales mencionados por los colaboradores se consideraron etnoespecies, es decir de acuerdo a la clasificación local (ver Zamudio \& Hilgert, 2015) y se ordenaron de acuerdo a los taxones botánicos científicos correspondientes para estas. Además, para corroborar los nombres científicos de los taxones, se utilizó la base de datos "Flora Argentina" del Instituto de Botánica Darwinion (2019).

\section{Resultados}

Las plantas silvestres comestibles y prácticas asociadas en las comunidades comechingonas de San Marcos Sierras

Uno de los entrevistados expresaba "todo lo ancestral (...) lo llevamos todos los hermanos; si te acostumbras a comer todo esto [plantas nativas] y dejas todo lo otro, estás vivo de verdad como los árboles, comiendo esto estás siempre fuerte, naturalmente como los árboles" (O.E.T., 56 años, San Marcos Sierras, 18/05/2017), dando cuenta del valor que tienen las plantas nativas en el bienestar cotidiano y la relación con su cultura.

Los entrevistados reconocieron 34 etnoespecies comestibles a través de sus nombres locales, con diversas formas de consumo. Éstas pertenecen a 16 familias botánicas, 26 géneros y 32 especies de la clasificación taxonómica científica (con 3 no identificadas a nivel de familia, 1 a nivel de género y 3 a nivel específico), (Tabla 1 y Fig. 2). Del total de etnoespecies, el $85 \%$ corresponde a taxones nativos y el resto a exóticos. Los "algarrobos" fueron las plantas citadas con mayor cantidad de partes utilizadas y formas de consumo. Entre ellos se incluyen a Prosopis alba Griseb., P. chilensis (Molina) Stuntz emend. Burkart, P. flexuosa DC. y P. nigra (Griseb.) Hieron. utilizados de igual manera en algunas recetas o distinguiéndolos en "algarrobo blanco" ( $P$. alba y P. chilensis) y "algarrobo negro" (P. nigra y P.flexuosa) para otras formas de consumo. 
Bol. Soc. Argent. Bot. 54 (2) 2019

Tabla 1. Especies vegetales silvestres comestibles reconocidas por los comechingones de San Marcos Sierras.

\begin{tabular}{|c|c|c|c|c|c|}
\hline Nombre Científico & $\begin{array}{l}\text { Nombre } \\
\text { local }\end{array}$ & Estatus & $\begin{array}{c}\text { Parte } \\
\text { utilizada }\end{array}$ & $\begin{array}{l}\text { Forma de } \\
\text { ingesta }\end{array}$ & $\begin{array}{c}\text { Formas de preparación } \\
\text { y observaciones }\end{array}$ \\
\hline \multicolumn{6}{|l|}{ Familia Anacardiaceae } \\
\hline $\begin{array}{l}\text { Lithraea molleoides } \\
\text { (Vell.) Engl. }\end{array}$ & molle & nativa & fruto & bebida & Como agregado del mate \\
\hline Schinus areira L. & aguaribay & nativa & resina & & $\begin{array}{l}\text { Se chupa como si fuera } \\
\text { un caramelo }\end{array}$ \\
\hline \multicolumn{6}{|l|}{ Familia Apocynaceae } \\
\hline $\begin{array}{l}\text { Araujia brachystephana } \\
\text { (Griseb.) Fontella \& Goyder } \\
\text { y A. odorata (Hook. \& } \\
\text { Arn.) Fontella \& Goyder }\end{array}$ & tasi o doca & nativa & fruto & fresco & $\begin{array}{l}\text { Cuando el fruto está inmaduro } \\
\text { se consume fresco }\end{array}$ \\
\hline \multicolumn{6}{|l|}{ Familia Arecaceae } \\
\hline $\begin{array}{l}\text { Trithrinax campestris } \\
\text { (Burmeist.) Drude \& Griseb. }\end{array}$ & $\begin{array}{l}\text { palma, } \\
\text { palma } \\
\text { caranday }\end{array}$ & nativa & fruto & fresco & $\begin{array}{l}\text { En el pasado, los niños } \\
\text { colectaban los frutos del suelo }\end{array}$ \\
\hline \multicolumn{6}{|l|}{ Familia Asteraceae } \\
\hline $\begin{array}{l}\text { Taraxacum officinale } \\
\text { F.H. Wigg., }\end{array}$ & $\begin{array}{l}\text { diente } \\
\text { de león }\end{array}$ & exótica & hoja & fresca & En ensalada \\
\hline \multicolumn{6}{|l|}{ Familia Boraginaceae } \\
\hline Symphytum officinale L. & confrey & exótica & hoja & fresca & $\begin{array}{l}\text { La ingieren las madres en } \\
\text { el período de lactancia }\end{array}$ \\
\hline \multicolumn{6}{|l|}{ Familia Brassicaceae } \\
\hline Rapistrum rugosum (L.) All. & mostacilla & exótica & hoja & $\begin{array}{l}\text { fresca } \\
\text { hervida }\end{array}$ & \\
\hline \multicolumn{6}{|l|}{ Familia Bromeliaceae } \\
\hline No identificada & chaguar & - & hoja & beber & $\begin{array}{l}\text { Se puede obtener agua de la hoja, } \\
\text { "se le saca primero una tirita" }\end{array}$ \\
\hline \multicolumn{6}{|l|}{ Familia Cactaceae } \\
\hline $\begin{array}{l}\text { Cereus forbesii Otto } \\
\text { ex C.F. Först. }\end{array}$ & ucle & nativa & fruto & $\begin{array}{l}\text { fresco } \\
\text { arrope }\end{array}$ & Se reconoce una colecta dificultosa \\
\hline $\begin{array}{l}\text { Cleistocactus baumannii } \\
\text { (Lem.) Lem. y Harrisia } \\
\text { pomanensis (F.A.C. Weber } \\
\text { ex K. Schum.) Britton \& Rose }\end{array}$ & chuli, ulúa & nativa & fruto & fresco & $\begin{array}{l}\text { "No es dulce". Se colecta con la } \\
\text { mano y se abre para consumir. }\end{array}$ \\
\hline \multirow[t]{2}{*}{$\begin{array}{l}\text { Opuntia ficus-indica (L.) Mill } \\
\text { f. ficus-indica y f. amyclaea }\end{array}$} & tuna & exótica & fruto & $\begin{array}{l}\text { licor } \\
\text { arrope } \\
\text { dulce } \\
\text { vinagre } \\
\text { vino }\end{array}$ & \\
\hline & & & cladodio & frito & \\
\hline Opuntia quimilo K. Schum & quimilo & nativa & cladodio & $\begin{array}{l}\text { hervida, } \\
\text { rebozada } \\
\text { y asada }\end{array}$ & $\begin{array}{l}\text { Se sacan previamente } \\
\text { las espinas y bordes. }\end{array}$ \\
\hline $\begin{array}{l}\text { Opuntia sulphurea } \\
\text { Gillies ex Salm-Dyck }\end{array}$ & tunilla & nativa & $\begin{array}{l}\text { cladodio } \\
\text { fruto }\end{array}$ & $\begin{array}{l}\text { frito } \\
\text { arrope }\end{array}$ & \\
\hline \multicolumn{6}{|l|}{ Familia Celtidaceae } \\
\hline \multirow[t]{2}{*}{$\begin{array}{l}\text { Celtis ehrenbergiana } \\
\text { (Klotzsch) Liebm. }\end{array}$} & tala & nativa & fruto & $\begin{array}{l}\text { fresco } \\
\text { con leche } \\
\text { licor }\end{array}$ & \\
\hline & & & hoja & fresca & \\
\hline
\end{tabular}




\section{Saur Palmieri y M. G. Geisa - Plantas comestibles en comunidades comechingonas}

Tabla 1. Continuación.

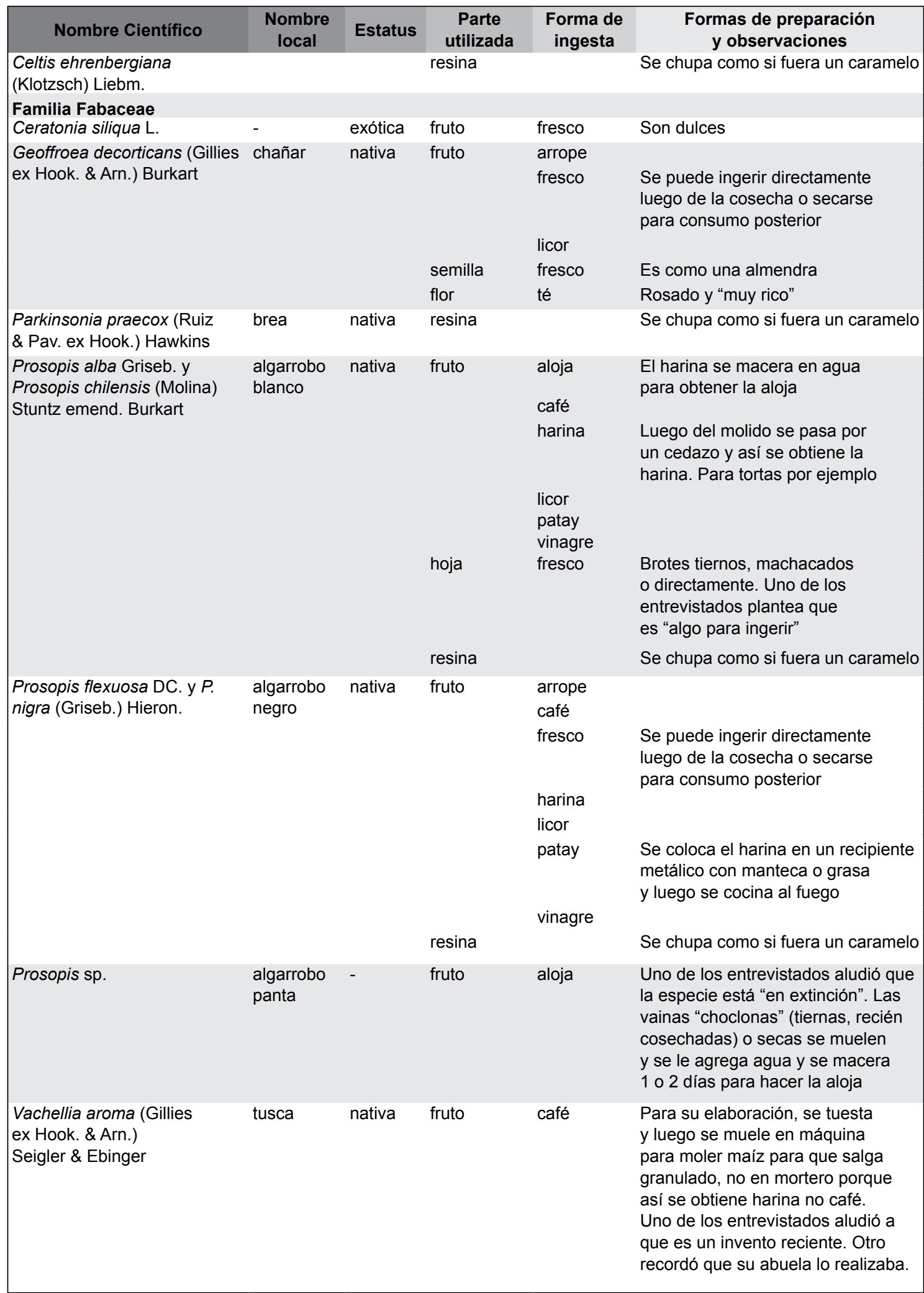


Bol. Soc. Argent. Bot. 54 (2) 2019

Tabla 1. Continuación.

\begin{tabular}{|c|c|c|c|c|c|}
\hline Nombre Científico & $\begin{array}{l}\text { Nombre } \\
\text { local }\end{array}$ & Estatus & $\begin{array}{c}\text { Parte } \\
\text { utilizada }\end{array}$ & $\begin{array}{l}\text { Forma de } \\
\text { ingesta }\end{array}$ & $\begin{array}{c}\text { Formas de preparación } \\
\text { y observaciones }\end{array}$ \\
\hline $\begin{array}{l}\text { Vachellia aroma (Gillies } \\
\text { ex Hook. \& Arn.) } \\
\text { Seigler \& Ebinger }\end{array}$ & & & & licor & \\
\hline $\begin{array}{l}\text { Vachellia caven (Molina) } \\
\text { Seigler \& Ebinger }\end{array}$ & espinillo & nativa & fruto & $\begin{array}{l}\text { café } \\
\text { tostadillo }\end{array}$ & $\begin{array}{l}\text { Un puñado de fruto tostado } \\
\text { en un jarrito de agua }\end{array}$ \\
\hline \multicolumn{6}{|l|}{ Familia Olacaceae } \\
\hline Ximenia americana L. & $\begin{array}{l}\text { albarillo } \\
\text { del campo }\end{array}$ & nativa & fruto & fresco & \\
\hline \multicolumn{6}{|l|}{ Familia Passifloraceae } \\
\hline Passiflora caerulea L. & $\begin{array}{l}\text { pasionaria, } \\
\text { granadilla }\end{array}$ & nativa & fruto & fresco & $\begin{array}{l}\text { El fruto se consume cuando } \\
\text { se encuentra inmaduro } \\
\text { "como un pepino" y cuando } \\
\text { madura como fruta fresca. }\end{array}$ \\
\hline \multicolumn{6}{|l|}{ Familia Plantaginaceae } \\
\hline Plantago sp. & llantén & - & hoja & fresca & $\begin{array}{l}\text { Se utiliza para elaborar ensaladas } \\
\text { junto a otras verduras }\end{array}$ \\
\hline \multicolumn{6}{|l|}{ Familia Polygonaceae } \\
\hline Rumex sp. & $\begin{array}{l}\text { lengua } \\
\text { de vaca }\end{array}$ & - & hoja & fresca & En ensalada \\
\hline \multicolumn{6}{|l|}{ Familia Rhamnaceae } \\
\hline $\begin{array}{l}\text { Condalia buxifolia Reissek } \\
\text { y C. microphylla Cav. }\end{array}$ & piquillín & nativa & fruto & $\begin{array}{l}\text { arrope } \\
\text { fresco } \\
\text { dulce } \\
\text { licor }\end{array}$ & $\begin{array}{l}\text { Formas de colecta: se coloca una } \\
\text { lona y luego se le pega a las ramas } \\
\text { o se sacuden para que caigan los } \\
\text { frutos; existe la creencia de que la } \\
\text { iguana hace lo mismo "con la cola". } \\
\text { También se colecta con la mano. } \\
\text { Los frutos se cocinan a } \\
\text { fuego directo revolviendo } \\
\text { de forma continua. }\end{array}$ \\
\hline $\begin{array}{l}\text { Sarcomphalus mistol } \\
\text { (Griseb.) Hauenschild }\end{array}$ & mistol & nativa & fruto & $\begin{array}{l}\text { bolanchao, } \\
\text { chunino, } \\
\text { pichico } \\
\\
\text { fresco } \\
\text { arrope } \\
\text { licor }\end{array}$ & $\begin{array}{l}\text { Se machaca en mortero el fruto } \\
\text { con agua y "se hace como una } \\
\text { gelatina", luego se realiza una } \\
\text { bolita que se pasa por harina de } \\
\text { harina de maíz molido tostado } \\
\text { (ankua) o en harina de algarroba. } \\
\text { Se seca con papel y se aprovecha } \\
\text { la harina que se desprende de las } \\
\text { "albóndigas". Esta golosina es muy } \\
\text { utilizada cuando se lleva las cabras } \\
\text { a pastar. Uno de los entrevistados } \\
\text { menciona que el nombre de la } \\
\text { preparación varía de acuerdo a } \\
\text { qué se emplee para rebozarla. }\end{array}$ \\
\hline \multicolumn{6}{|l|}{ Familia Solanaceae } \\
\hline Capsicum chacoense Hunz. & $\begin{array}{l}\text { ají de } \\
\text { monte, } \\
\text { quitucho }\end{array}$ & nativa & fruto & condimento & Fresco o seco \\
\hline
\end{tabular}




\section{Saur Palmieriy M. G. Geisa - Plantas comestibles en comunidades comechingonas}

Tabla 1. Continuación.

\begin{tabular}{|c|c|c|c|c|c|}
\hline Nombre Científico & $\begin{array}{l}\text { Nombre } \\
\text { local }\end{array}$ & Estatus & $\begin{array}{c}\text { Parte } \\
\text { utilizada }\end{array}$ & $\begin{array}{l}\text { Forma de } \\
\text { ingesta }\end{array}$ & $\begin{array}{c}\text { Formas de preparación } \\
\text { y observaciones }\end{array}$ \\
\hline \multirow[t]{2}{*}{$\begin{array}{l}\text { Salpichroa origanifolia } \\
\text { (Lam.) Baill. }\end{array}$} & $\begin{array}{l}\text { uvilla, } \\
\text { uvita del } \\
\text { campo }\end{array}$ & nativa & flor & fresco & Como golosinas \\
\hline & & & fruto & fresco & Como golosinas \\
\hline \multicolumn{6}{|l|}{ Familia Verbenaceae } \\
\hline $\begin{array}{l}\text { Lippia integrifolia } \\
\text { (Griseb.) Hieron }\end{array}$ & incayuyo & nativa & hoja & fresca & \\
\hline \multicolumn{6}{|l|}{ Familias no identificadas } \\
\hline No identificada & bulbos & - & $\begin{array}{l}\text { órgano } \\
\text { subterráneo }\end{array}$ & - & \\
\hline No identificada & ortiga & - & $\begin{array}{l}\text { toda la } \\
\text { planta, } \\
\text { menos } \\
\text { la raíz }\end{array}$ & sopa & $\begin{array}{l}\text { Se puede combinar con } \\
\text { otras verduras }\end{array}$ \\
\hline No identificada & - & - & flor & fresco & $\begin{array}{l}\text { Cuando niño chupaban la flor de } \\
\text { una planta similar a Lonicera }\end{array}$ \\
\hline
\end{tabular}

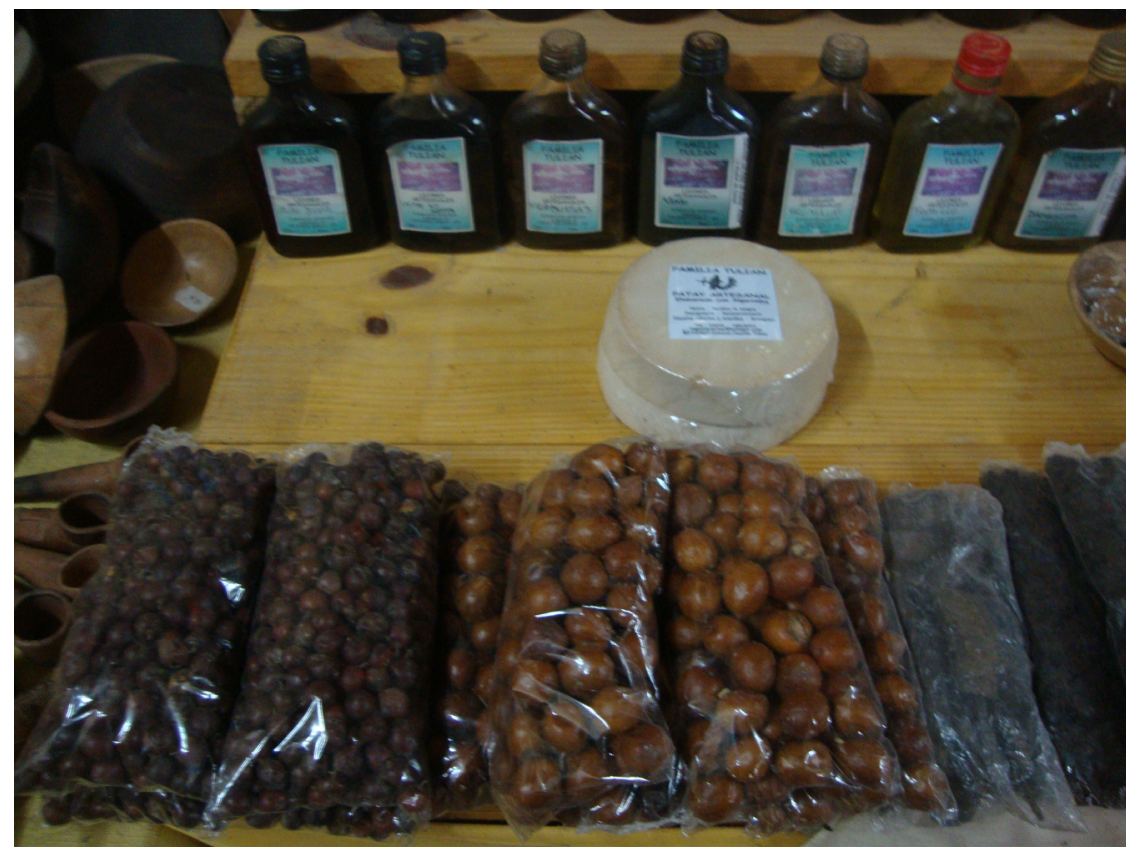

Fig. 2. Productos elaborados con frutos comestibles. Se pueden observar licores, patay y frutos envasados para la venta.

En segundo lugar, se menciona el consumo del "tala" (Celtis erhenbergiana (Klotzsch) Liebm.) a través de sus frutos y hojas frescas, seguido del "chañar" (Geoffroea decorticans (Gillies ex Hook. \& Arn.) Burkart), el "mistol" (Sarcomphalus mistol (Griseb.) Hauenschild) y el "piquillín" (Condalia buxifolia Reissek y C. microphylla Cav.).
Las partes vegetales más utilizadas son los frutos, pero también se mencionaron hojas, cladodios, resinas y flores. Uno de los entrevistados recordó que un conocido en la zona de Pichanas (Dpto. Cruz del Eje) consumía "bulbos", aludiendo a partes vegetativas subterráneas, aunque no recuerda qué preparaciones realizaba. Sin embargo, otro 
entrevistado afirmó que no se consumen las "raíces" de ninguna planta; sólo se da cuenta de su utilización en el pasado en momentos antes del fallecimiento de una persona, "para transitar el vacio de la muerte". Con respecto a las resinas, se mencionó que se sorbe "el lloro" de Prosopis spp., "brea” (Parkinsonia praecox (Ruiz \& Pav. ex Hook.) Hawkins), "aguaribay" (Schinus areira L.) y "tala".

En las tres entrevistas, se observó que las personas mencionaban de forma espontánea, usos medicinales relacionados con las plantas alimenticias que citaban, expresando ejemplos de los beneficios que les atribuyen para la salud. A los fines de este trabajo sólo hemos citado los usos alimenticios, concentrándonos en los objetivos propuestos.

La colecta de frutos nativos se practica poco en el presente, según lo que los entrevistados refieren. Cuando se realiza, el destino de los productos elaborados es el consumo familiar o la venta. Por ejemplo, uno de los entrevistados ofrece los frutos envasados y productos derivados al turismo (Fig. 2).

En relación a la colecta, los frutos de los "algarrobos" (Prosopis spp.) se pueden levantar del suelo o se pueden tomar directamente de la planta. Mientras que los de "piquilín" (Condalia spp.) se obtienen a partir de la colocación de una lona bajo el vegetal y/o con el agitado o golpeteo de las ramas. Por otro lado, se utiliza algún instrumento en el caso de cactáceas para evitar las espinas. Uno de los entrevistados cita que "se colecta en los lugares donde crecen los yuyos" aludiendo a que deben movilizarse para buscar la planta de interés en sitios diferentes según el caso (por ejemplo, cerca del río o en las sierras). Algunas crecen alrededor de la casa y otros se buscan en lugares que ahora son privados y en donde deben pedir permiso para ingresar o en el monte.

Como formas de conservación se citó la utilización de un depósito construido con ramas de "jarilla" (Larrea divaricata L.) denominado pirgua al que se le agrega tierra y "matapulgas" (Schkuhria pinnata (Lam.) Kuntze ex Thell.) o "atamisqui" (Atamisquea emarginata Miers ex Hook. \& Arn.) que ayuda a ahuyentar las polillas. De esta manera se pueden conservar las vainas de Prosopis spp. El añadido de ceniza también se citó como forma de ahuyentar insectos. Además, se mencionaron la molienda, tostado y el patay como formas de conservar algunos alimentos. Los frutos de "chañar", "mistol" y "algarrobos" se pueden conservar en canastos realizados con la fibra de "palma caranday" (Trithrinax campestris (Burmeist.) Drude \& Griseb.), "porque tiene ponzoña que aleja el bicho, y tiene la entrada de aire justo" que evita la formación de polillas.

Las cinco especies exóticas y sus usos mencionados son los siguientes: la "tuna" (Opuntia ficus-indica (L.) Mill (Cactaceae) en sus dos formas: ficus-indica y amyclaea) de la cual se utiliza el fruto maduro para elaborar licor, arrope, dulce, vinagre y vino y los cladodios que pueden freírse cuando son pequeños. Además, se indicó la posibilidad de realizar ensaladas con las hojas de "diente de león" (Taraxacum officinale F.H. Wigg., Asteraceae). Se aprovechan asimismo las vainas dulces de Ceratonia siliqua L. (Fabaceae) y la planta de "mostacilla" (Rapistrum rugosum (L.) All., Brassicaceae) fresca o hervida. Por último, se mencionó el "confrey" (Symphytum officinale L., Boraginaceae) que se ingiere en el período de lactancia.

\section{Discusión}

En la localidad de San Marcos Sierras, desde los años 1990, y en consonancia con la coyuntura de reemergencia étnica de Córdoba (Bompadre, 2013), viene ocurriendo un proceso de reconocimiento de muchas personas como pertenecientes a comunidades originarias. En este contexto, resulta interesante conocer cuáles son los saberes que los autodenominados comechingones poseen, en particular sobre las plantas comestibles. En este sentido, en este primer acercamiento a las comunidades, podemos concluir que dichos pobladores de San Marcos Sierras conocen un gran número de plantas comestibles en comparación con la mayoría de los trabajos realizados en el sector serrano de la provincia con otros grupos sociales. Por ejemplo, Arias Toledo (2008) 19 especies, Arias Toledo et al. (2007a) citan 13 especies, Martínez et al. (2016) 6 especies, Trillo et al. (2014) 15 especies. El número hallado en el presente trabajo, es elevado respecto de los mencionados anteriormente, y similar a la suma relevada por Fernández Marinaro \& Martínez (2019) quienes contabilizaron 34 especies 


\section{Saur Palmieri y M. G. Geisa - Plantas comestibles en comunidades comechingonas}

vegetales alimenticias. El género Prosopis fue el más citado por los entrevistados, coincidiendo con Scarpa (2009) sobre el uso de plantas comestibles que realizan numerosos pueblos originarios en la región del Gran Chaco. Muchas de las especies $\mathrm{y}$ formas de consumo citada en este estudio, han sido mencionadas en trabajos previos del sector serrano cordobés -i.e. frutos de "chañar", "mistol" y "algarrobos" en arrope y consumo directo, "ají del monte" como condimento, "molle de beber" como saborizante del mate, "lengua de vaca" en ensalada (ver Saur Palmieri et al., 2018, Fernández Marinaro \& Martínez, 2019). En relación al consumo de estructuras florales, es breve el registro de su aprovechamiento. Fernández Marinaro \& Martínez (2019) enumeran el consumo de flores de Robinia pseudoacacia L. (Fabaceae), Passiflora spp. (Passifloraceae) y el polen de Bidens pilosa L. (Asteraceae) como azafrán. Por ende, este estudio representa el primer antecedente de la utilización como alimento de flores de "chañar" y "uvita del campo". Por otro lado, no se había registrado con anterioridad la ingesta con fines alimenticios de hojas de especies arbóreas ("tala" y "algarrobos"), resinas como golosina ("tala", "brea", "aguaribay", "algarrobos", "chañar") y órganos subterráneos en la provincia de Córdoba. Existen antecedentes de la utilización de estas partes vegetales en comunidades originarias del Gran Chaco, y en la etnia Toba del Impenetrable Chaqueño (Argentina) coincidiendo en que los frutos son el ítem utilizado en mayor proporción e incorporando además los órganos subterráneos (Arenas \& Scarpa, 2007; Scarpa, 2009; Martínez, 2012); sin embargo, no se registra la ingesta de resinas en trabajos previos. Respecto a los órganos subterráneos enumerados, se requiere profundizar el estudio para identificar los taxones referidos.

Además, pudimos observar, aunque requiere de estudios más precisos, saberes de diverso proceder que componen el conocimiento sobre plantas comestibles del grupo social mencionado. Por un lado, la tradición oral podría ser muy importante en cuanto a que ha sido mencionada, sobre todo cuando proviene de parientes en línea ascendente. Por ejemplo, se recuerda que en su infancia, los abuelos llevaban al campo a los niños y les hacían probar distintos alimentos y medicinas. En este sentido, uno de los informantes recuerda cómo su abuela agradecía a la Pachamama cuando tomaba una hoja de "tala" o "mistol" para consumir. Un entrevistado mencionó: "soy de una época en que los padres eran humildes, simples (...) y se hablaba" (R. V., 71 años, San Marcos Sierras, 18/05/2017), aludiendo a la importancia que tenía, sobre todo en el pasado, la tradición oral. Por otro lado, el contacto con otros (charlas, cursos, etc. que han sido aludidos en las entrevistas), resultaría en un intercambio de conocimientos que podría aportar en lo que las personas entienden sobre plantas comestibles. Por último, las fuentes escritas también contribuirían a lo mencionado, ya que se pudo observar la existencia en las casas de los entrevistados de libros sobre vegetales. A futuro, y considerando la diversidad de fuentes que de conocimiento que pudieron observarse en este trabajo exploratorio, surge la necesidad de profundizar el estudio de este aspecto a fin de aproximarnos al origen de los saberes sobre las plantas y formas de ingesta mencionadas, calendario y formas de colecta en cada caso. Además, se sugiere complementar la información obtenida en base a la identificación botánica mediante catálogo fotográfico con salidas a campo de reconocimiento.

Por otro lado, podemos visualizar los saberes de este Pueblo, profundamente arraigados al Chaco Serrano cordobés, como una herramienta fundamental en el proceso de reconocimiento comunitario y reivindicación territorial. Asimismo, considerando la noción de Soberanía Alimentaria determinamos que la recolección de vegetales nativos del bosque nativo constituye una práctica cultural vigente. Si bien el grado de importancia que esta actividad posee, requiere mayores análisis, consideramos que esta práctica permitiría a las personas decidir activamente su propio sistema de alimentación, accediendo a alimentos sanos y arraigados culturalmente. Igualmente, si se tiene cuenta que los entrevistados refirieron el uso medicinal de ciertos taxones en simultáneo al empleo comestible, deriva que la soberanía alimentaria está indisolublemente ligada a la sanitaria. Se destaca así el rol del bosque nativo en la alimentación y salud soberanas y el sustento de las culturas.

En general, los trabajos preexistentes acerca del empleo de plantas nativas con fines alimenticios en el noroeste de Córdoba (Trillo et al., 2014; Torrico Chalabe \& Trillo, 2015; Trillo, 2016; Arias 
Toledo et al., 2007a, 2007b; Grimaldi \& Trillo, 2018; Fernández Marinaro \& Martínez, 2019), hacen referencia a comunidades campesinas, sin contar con antecedentes de trabajos relacionados con personas autodenominadas originarias en la provincia. Comparando los vegetales mencionados en cada estudio, se citan nuevos registros en este trabajo, como la "brea" y el "incayuyo", que complementan las investigaciones precedentes. Consideramos en este sentido, que es de relevancia profundizar las indagaciones en instancias futuras, acerca de las formas de consumo, colecta y procesamiento a los fines de complementar los saberes culturales observados en las comunidades comechingonas.

Esperamos que este trabajo constituya un aporte a la continuidad de la cultura de este Pueblo, a su visibilización y a la difusión de sus saberes ancestrales utilizados en la actualidad.

\section{Conclusiones}

Este trabajo ofrece una primera aproximación acerca de las plantas empleadas por los pueblos originarios de Córdoba. Aunque, es necesario profundizar este abordaje en futuras instancias, se pudieron evidenciar conocimientos de importancia ecológica y cultural a nivel local y provincial, que no tenían antecedentes en la Provincia de Córdoba. Los integrantes de las comunidades comechingonas de San Marcos Sierras incorporan en sus dietas una amplia variedad de plantas nativas comestibles a partir de diferentes partes vegetales y formas de consumo. Consideramos que la asociación que mantienen con las plantas nativas de su ambiente es un hecho de relevancia que puede contribuir en futuras estrategias de conservación de los ecosistemas de la zona, a la reinvindicación de la cultura Comechingón y al desarrollo de sistemas de alimentación soberanos de estos pueblos.

\section{Contribución de los autores}

Ambas autoras han realizado conjuntamente y a partes iguales la colecta de datos, su interpretación y redacción del manuscrito. VSP confeccionó las figuras.

\section{Agradecimientos}

Agradecemos profundamente a Raúl Verasay y Nelly de Verasay (Comunidad Orko Huasi), Oscar Eduardo "Meliño" Tulián y María Celeste Bordagaray (Comunidad Tulián) y Leopoldo Tulián (Comunidad Tacu Kuntur) por colaborar con este trabajo. Asimismo, agradecemos las contribuciones de los revisores anónimos y la editora Norma Hilgert que posibilitaron la mejora sustancial de este trabajo. Los conocimientos locales referidos en este trabajo pertenecen a las comunidades de San Marcos Sierras, Córdoba. Las autoras agradecen a CONICET y al CIN, que financiaron parcialmente este trabajo y a la Universidad Nacional de Córdoba que proveyó espacio físico y material bibliográfico para su realización.

\section{Bibliografía}

ADIC (ASOCIACIÓN DE DESCENDIENTES INDÍGENAS DE CÓRDOBA). 2000. San Marcos Sierras. Reseña histórica. Disponible en: https:// www.sanmarcossierras.com/historia.htm [Acceso: 20 de mayo de 2017].

AGUIRRE, M. G. 2012. Recursos vegetales: uso, consumo y producción en la Puna meridional argentina (5000-1500 AP). Tesis de Doctorado. Facultad de Ciencias Naturales y Museo. Universidad Nacional de La Plata. Buenos Aires, Argentina.

ALDAZABAL, V., E. EUGENIO \& D. N. ÁVIDO. 2012. Indagando en los hábitos alimenticios de los cazadores recolectores de la Pampa Deprimida. En: Universidad Nacional de Córdoba (ed.), Las manos en la masa. Arqueologías, Antropologías e Historias de la Alimentación en Sudamérica, pp. 425-441. Córdoba, Argentina.

ARENAS, P. A. 2003. Etnografía y alimentación entre los Toba-Ñachilamole\#ek y Wichi-Lhuku'tas del Chaco Central (Argentina). Buenos Aires, Argentina.

ARENAS, P. \& G. F. SCARPA. 2007. Edible wild plants of the Chorote Indians, Gran Chaco, Argentina. Bot. J. Linn. Soc. 153: 73-85.

ARIAS TOLEDO, B. 2008. Disponibilidad y uso de las plantas silvestres alimenticias y medicinales en las Sierras de Córdoba: su asociación con factores fitogeográficos y culturales. Tesis Doctoral en Ciencias Biológicas. Facultad de Ciencias Exactas, Físicas y Naturales. Universidad Nacional de Córdoba. Córdoba, Argentina.

ARIAS TOLEDO, B., S. COLANTONIO \& L. GALETTO. 2007a. Knowledge and use of edible and 


\section{Saur Palmieri y M. G. Geisa - Plantas comestibles en comunidades comechingonas}

medicinal plants in two populations from the Chaco forest, Córdoba province, Argentina. J. Ethnobiol. 27: 218-232. https://doi.org/10.2993/02780771(2007)27[218:KAUOEA]2.0.CO;2

ARIAS TOLEDO, B., L. GALETTO \& S. COLANTONIO. 2007b. Uso de plantas medicinales y alimenticias según características socioculturales en Villa Los Aromos (Córdoba, Argentina). Kurtziana 33: 79-88

AZAR, F. P. 2002. Utilización de vegetales en las sociedades indigenas norpatagónicas, contribución a una base de datos v.1. Tesis. Facultad de Filosofía y Letras. Universidad de Buenos Aires. Buenos Aires, Argentina.

BARTOLOMÉ, M. A. 1997. Gente de costumbre y gente de razón: las identidades étnicas en México. Siglo XXI. Ciudad de México, México.

BERNARD, H. R. 1995. Research Methods in Anthropology Qualitative and Quantitative Approaches. Altamira Press, Estados Unidos de América.

BIURRUN, E., L. GALETTO, A. M. ANTON \& F. BIURRUN. 2007. Plantas silvestres comestibles utilizadas en poblaciones rurales de la Provincia de La Rioja (Argentina). Kurtziana 33: 121-140.

BOMPADRE, J. M. 2013. Procesos de comunalización contemporánea de pueblos originarios en contextos urbanos y rurales de la provincia de Córdoba. X Reunión de Antropología del Mercosur, Universidad Nacional de Córdoba, Argentina.

BRIONES, C. 1998. La alteridad del "cuarto mundo”. Una deconstrucción antropológica de la diferencia. Serie Antropológica. Ediciones del Sol. Buenos Aires, Argentina.

CABRERA, A. L. 1976. Regiones fitogeográficas argentinas. En: ACME (ed.) Enciclopedia Argentina de Agricultura y Jardinería, pp. 1-85. Buenos Aires, Argentina.

CANO-CONTRERAS, E. J., A. MEDINACELI, O. L. SANABRIA-DIAGO \& A. ARGUETA. 2016. Código de ética para la investigación, la investigación-acción y la colaboración etnocientífica en América Latina. Etnobiología 14: 1-32.

COTTON, C. M. 1996. Ethnobotany: principles and applications. John Wiley \& Sons. Londres, Inglaterra.

CULTURA SAN MARCOS SIERRAS. 2017. Encuentro de Familias de Origen Comechingón 2017. Disponible en: https://www. facebook.com/culturadesanmarcossierras/ posts/1243803759066113 [Acceso: 22 mayo de 2017].

DE SOUSA SANTOS, B. 2010. Para descolonizar Occidente. Más allá del pensamiento abismal. CLACSO, Buenos Aires, Argentina.

DI LULLO, O. 1935. La alimentación popular de Santiago del Estero. Talleres Gráficos El Literal. Santiago del Estero.
FERNÁNDEZ MARINARO, A. \& G. J. MARTÍNEZ. 2019. Las plantas en la alimentación de pobladores rurales de los ambientes serranos de La Calera (Dpto. Colón, Córdoba, Argentina). Una perspectiva etnobotánica diacrónica. Bonplandia 28: 43-69.

http://dx.doi.org/10.30972/bon.2813573

FORO MUNDIAL DE LA SOBERANÍA ALIMENTARIA. 2007. Declaración de Nyéléni. Disponible en: https://nyeleni.org/spip. php?article291 [Acceso: 12 diciembre de 2018].

GIORGIS, M. A., A. M. CINGOLANI, F. CHIARINI, J. CHIAPELLA, G. BARBOZA, L. ARIZA ESPINAR, R. MORERO, D. E. GURVICH, P. A. TECCO, R. SUBILS \& M. CABIDO. 2011. Composición florística del Bosque Chaqueño Serrano en la provincia de Córdoba, Argentina. Kurtziana 36: 9-43.

GLESER, A. 2009. Los hijos del Suquia. Los Comechingones del Pueblo de la Toma, actual barrio Alberdi, ayer y hoy. Ed. Imprentica, Córdoba.

GRIMALDI, P. A. \& C. TRILLO. 2018. Prácticas y usos tradicionales de "cerco" y "monte" por los criollos de San Marcos Sierras (Córdoba, Argentina). Bonplandia 27: 81-97.

http://dx.doi.org/10.30972/bon.2712986

HILGERT, N. I. 1999. Las plantas comestibles en un sector de las Yungas meridionales (Argentina). Anales Jard. Bot. Madrid. 57: 117-138.

HURRELL, J., M. POCHETTINO, J. PUENTES \& P. ARENAS. 2013. Del marco tradicional al escenario urbano: Plantas ancestrales devenidas suplementos dietéticos en la conurbación Buenos Aires-La Plata, Argentina. BLACPMA 12: 499-515.

INDEC (INSTITUTO NACIONAL DE ESTADÍSTICA Y CENSOS). 2004. Encuesta Complementaria de Pueblos Indígenas [base de datos en línea]. Disponible en: http://www.indec.gov.ar/micro sitios/webcenso/ECPI/index_ecpi.asp [Acceso: 12 mayo de 2017].

INDEC (INSTITUTO NACIONAL DE ESTADÍSTICA Y CENSOS). 2010. Censo Nacional de Población, Hogares y Viviendas. Argentina [base de datos en línea]. Disponible en: www.indec.gov.ar [Acceso: 12 mayo de 2017].

INSTITUTO DE BOTÁNICA DARWINION. 2019. Base de datos "Flora Argentina". Disponible en: http://www.floraargentina.edu.ar [Acceso: 23 de enero 2019].

LADIO, A. 2004. El uso actual de plantas nativas silvestres y comestibles en poblaciones mapuches del NO de la Patagonia. BLACPMA 3: 30-34.

LADIO, A. 2005. Malezas exóticas comestibles y medicinales utilizadas en poblaciones del Noroeste patagónico: aspectos etnobotánicos y ecológicos. BLACPMA 4: 75. 
LADIO, A. H. \& M. LOZADA. 2000. Edible wild plant use in a Mapuche community of northwestern Patagonia. Human Ecol. 28: 53-71.

LADIO, A. \& E. H. RAPOPORT. 1999. El uso de plantas silvestres comestibles en una población suburbana del noroeste de la Patagonia. Parodiana 11: 49-62.

LEY PROVINCIAL N¹0.308. 2015. Boletín Oficial de la Provincia de Córdoba del 21 de octubre. Córdoba, Argentina. Disponible en: http:// boletinoficial.cba.gov.ar/wp-content/4p96humu zp/2015/10/21102015_BOCba_1sNRmb2D.pdf [Acceso 13 mayo de 2017).

LÓPEZ, M. L. 2015. La cocina como medio para la reproducción social de los grupos prehispánicos de las sierras de Córdoba. En: J. Salazar (ed.) Condiciones de Posibilidad de la Reproducción Social en Sociedades Prehispánicas y Coloniales Tempranas en las Sierras Pampeana (República Argentina), pp. 177-212. Centro de Estudios Históricos "Prof. Carlos S. A. Segreti", Córdoba, Argentina.

MARTÍNEZ, G. J. 2012. Actualidad y pasado del uso de plantas silvestres comestibles entre los Tobas del Impenetrable chaqueño - Reflexiones, discursos y prácticas en torno a la alimentación. En: Universidad Nacional de Córdoba (ed.) Las manos en la masa. Arqueologías, Antropologías e Historias de la Alimentación en Sudamérica, pp.47-69. Córdoba, Argentina.

MARTÍNEZ CROVETTO, R. N. 2012. Estudios Etnobotánicos V. Nombres de plantas y su utilidad según los Mbya Guaraní de Misiones, Argentina. Bonplandia 21: 109-133.

MARTÍNEZ, G., C. ROMERO, C. PEN, M. VILLAR \& P. DURANDO. 2016. Etnobotánica participativa en escuelas rurales de la Comuna Paso Viejo (Departamento Cruz del Eje, Córdoba, Argentina). Bonplandia 25: 145-162.

MEDINA, M. E., S. PASTOR \& A. RECALDE. 2016. The archaeological landscape of Late Prehispanic mixed foraging and cultivation economy (Sierras of Córdoba, Argentina). J. Anthropol. Archaeol. 42: 88-104.

MINISTERIO DE EDUCACIÓN \& UNICEF. 2010. CEAPI. Disponible en: http://www.bnm.me.gov. ar/giga1/documentos/EL002883.pdf [Acceso: 10 mayo de 2017].

MONTANI, M. C. \& G. F. SCARPA. 2016. Recursos vegetales y prácticas alimentarias entre indígenas Tapiete del Noroeste de la Provincia de Salta, Argentina. Darwiniana 4: 12-30.

MUIÑO, W. 2012. Estudio etnobotánico de plantas usadas en la alimentación de los campesinos del noroeste de la Pampa argentina. Chungara 44: 389-400.
MUNICIPALIDAD DE SAN MARCOS SIERRAS \& UNIVERSIDAD NACIONAL DE CÓRDOBA. 2009. Cuadernillo de Ordenanza de Planeación y Ordenamiento territorial $N^{\circ} 596 / 09$. Primera etapa. Ordenanza de ampliación de emergencia hídrica N595/09. Plan de Trabajo Talleres de Planificación local. Segunda etapa 2010. Municipio de San Marcos Sierras y Escuela de Trabajo Social - UNC. Córdoba, Argentina.

OCHOA, J. J. \& A. H. LADIO. 2011. Plantas silvestres con órganos subterráneos comestibles: transmisión cultural sobre recursos subutilizados en la Patagonia (Argentina). BLACPMA 14: 287-300.

PALLADINO, L. 2013. Usos del pasado territorial en el proceso de comunalización de los Comechingones del Pueblo de La Toma, Córdoba, Argentina. Corpus 3: 1-25. https://doi.org/10.4000/corpusarchivos.512

PASTOR, S. \& L. LÓPEZ. 2011. Consideraciones sobre la agricultura prehispánica en el sector central de las Sierras de Córdoba. En: Magma (ed.) Arqueología de la Agricultura: Casos de Estudio en la Región Andina Argentina, pp. 208-233. Tucumán, Argentina.

PASTOR, S., M. MEDINA, A. RECALDE, L. LÓPEZ \& E. BERBERIÁN. 2012. Arqueología de la región montañosa central de Argentina. Avances en el conocimiento de la Historia Prehispánica Tardía. Relaciones 37: 89-112.

RAPOPORT, E. H., A. LADIO, E. RAFFAELE, L. GHERMANDI \& E. H. SANZ. 1998. Malezas comestibles. Hay yuyos y yuyos... Rev. de la Asociación Científica y Tecnológica de la Asociación Ciencia Hoy 9 (49).

RAPOPORT, E. H., A. MARZOCCA \& B. S. DRAUSAL. 2009. Malezas comestibles del Cono Sur y otras partes del planeta. INTA, Argentina.

RÍO, M. \& L. ACHÁVAL. 1905. Geografia de la Provincia de Córdoba, Volumen II. Gobierno de la Provincia de Córdoba, Argentina.

ROSSET, P. 2003. Food Sovereignty: Global Rallying Cry of Farmer Movements. Food First Backgrounder 9: 1-4.

SAUR PALMIERI, V., M. L. LÓPEZ \& C. TRILLO. 2018. Aproximaciones etnobotánicas de las especies y prácticas de frutos nativos comestibles de la actualidad. Aportes para la interpretación del pasado prehispánico de Cerro Colorado (Córdoba, Argentina). Bol. Soc. Argent. Bot. 53: 115-133.

SCARPA, G. F. 2009. Wild food plants used by the indigenous people of the South American Gran Chaco: A general synopsis and intercultural comparison. $J$. Appl. Bot. Food Qual. 83: 90-101.

STAGNARO, M. 2009. Política y movimiento indígena en Córdoba. Imaginarios, comunidades e instituciones en la (re)emergencia indígena local. En: UNSAM, IDAES (eds.) VIII Reunión de Antropología del Mercosur "Diversidad y Poder en América Latina". Buenos Aires, Argentina. 


\section{Saur Palmieri y M. G. Geisa - Plantas comestibles en comunidades comechingonas}

STAGNARO, M. 2016. Pueblos indígenas en Santiago del Estero y Córdoba. En: Ministerio de Educación y Deportes Presidencia de la Nación (ed.) Pueblos indigenas en la Argentina, historias, culturas, lenguas y educación. Ciudad Autónoma de Buenos Aires, Argentina.

TORRICO CHALABE, J. K. \& C. TRILLO. 2015. Prácticas de manejo, usos y valoración de taxones de Cactaceae en el noroeste de Córdoba, Argentina. Bonplandia 24: 5-22.

TRILLO, C. 2010. Valoración del Bosque y Uso de las Plantas Silvestres por Parte de los Pobladores del Valle de Guasapampa, Noroeste de la Provincia de Córdoba. Tesis doctoral, Facultad de Ciencias Exactas Físicas y Naturales, Universidad Nacional de Córdoba, Córdoba, Argentina.

TRILLO, C. 2016. Prácticas tradicionales de manejo de recursos vegetales en unidades de paisajes culturales del oeste de la provincia de Córdoba, Argentina. Zonas áridas 16: 86-111. http://dx.doi.org/10.21704/za.v16i1.640
TRILLO, C., S. COLANTONIO \& L. GALETTO 2014. Perceptions and Use of Native Forests in the Arid Chaco of Córdoba, Argentina. Ethnobotany Research \& Applications 12: 497-510.

TULIÁN, M. J. 2016. Zoncoipacha: desde el corazón del Territorio, el legado de Francisco Tulián. Ciccus. Buenos Aires, Argentina.

TULIÁN, M. J. \& L. PALLADINO. 2015. Revivir el territorio sagrado. A los pasos de Francisco Tulián. Cardinalis 5: 232-255.

VALVERDE, S. 2013. De la invisibilización a la construcción como sujetos sociales: el pueblo indígena Mapuche y sus movimentos en Patagonia, Argentina. Anuário Antropológico: 139-166. https://doi.org/10.4000/aa.414

ZAMUDIO, F. \& N. HILGERT. 2015. Multidimensionality and variability in folk classification of stingless bees (Apidae: Meliponini). Journal of Ethnobiology and Ethnomedicine 11: 41. https://doi.org/10.1186/s13002-015-0029-z 
